\title{
No Chattering and Adaptive Sliding Mode Control of a Fractional-Order Phase Converter with Disturbances and Parameter Uncertainties
}

\author{
Karthikeyan Rajagopal (iD, Riessom Weldegiorgis, Anitha Karthikeyan, Prakash Duraisamy, \\ and Goitom Tadesse
}

Center for Nonlinear Dynamics, Defence University, Ethiopia

Correspondence should be addressed to Karthikeyan Rajagopal; rkarthiekeyan@gmail.com

Received 16 March 2018; Revised 27 July 2018; Accepted 31 July 2018; Published 22 October 2018

Academic Editor: Lucia Valentina Gambuzza

Copyright (C) 2018 Karthikeyan Rajagopal et al. This is an open access article distributed under the Creative Commons Attribution License, which permits unrestricted use, distribution, and reproduction in any medium, provided the original work is properly cited.

\begin{abstract}
Discussing the dynamical properties of various power system models is of significant importance in order to understand its complete behavior. Even though there are many literatures discussing about the chaotic behavior shown by phase converter circuits, none of them have reported the hazardous phenomenon of multistability. In this paper, we derive the fractional-order model of a phase converter circuit and investigate its dynamics. Bifurcation of the system with the parameters and fractional order are investigated. A forward and backward continuation scheme is adopted to display various coexisting attractors; the property of multistability is also discussed. Using forward and backward continuation, various coexisting attractors and the property of multistability are discussed. Two different sliding mode controllers for controlling chaotic oscillations with model disturbances and parameter uncertainties are derived, and the effectiveness of the controllers is discussed with numerical simulations.
\end{abstract}

\section{Introduction}

The nonlinear dynamical systems are described using nonlinear differential equations and represented in state (phase) space. The system state in steady state can be defined by equilibrium point and by limit cycles in state space; when the system is subjected to aperiodic oscillations, the system can be in quasiperiodic or in chaotic state. For different parameter values of a nonlinear system, the location and the number of equilibrium points can change. According to Lyapunov, regular attractors (steady states, limit cycles) are fully stable or fully unstable, but this is not true for chaotic attractors. Deterministic chaos is a phenomenon when small discrepancies in the initial conditions lead to an unpredictable behavior. The average rate of expansion along the principle axes are known as Lyapunov exponents. The presence of at least one positive Lyapunov exponent confirms chaos in the system. The chaotic behavior in electrical drive systems are noted as undesirable.
Most of the power systems are composed of ordinary differential equations; the system state changes (drastic changes in current or voltage decreasing or increasing rate) lead to the nonlinear dynamical behavior and makes the circuit modeling complex [1]. The last two decades have witnessed multiple literatures discussing about the chaotic behavior of various power system models. To be specific, three cases, (i) high-frequency, time-sharing inverter, (ii) hysteresis current-controlled three-phase voltage source converter, and (iii) dual-channel resonant converter, were studied for existence and effectiveness of chaotic behavior in power electronics [2]. Nonlinear behaviors, especially chaotic phenomena of the three-phase voltage source inverter (VSI) with hysteresis current comparator, are studied [3]. Chaotic behavior of various types of switching converters is discussed in [2,4-7]; the analytical model of switching converters with piecewise switched circuits is developed during the 1970s. The changes in the switching state in power converters results in nonsmooth system trajectories, and hence, 


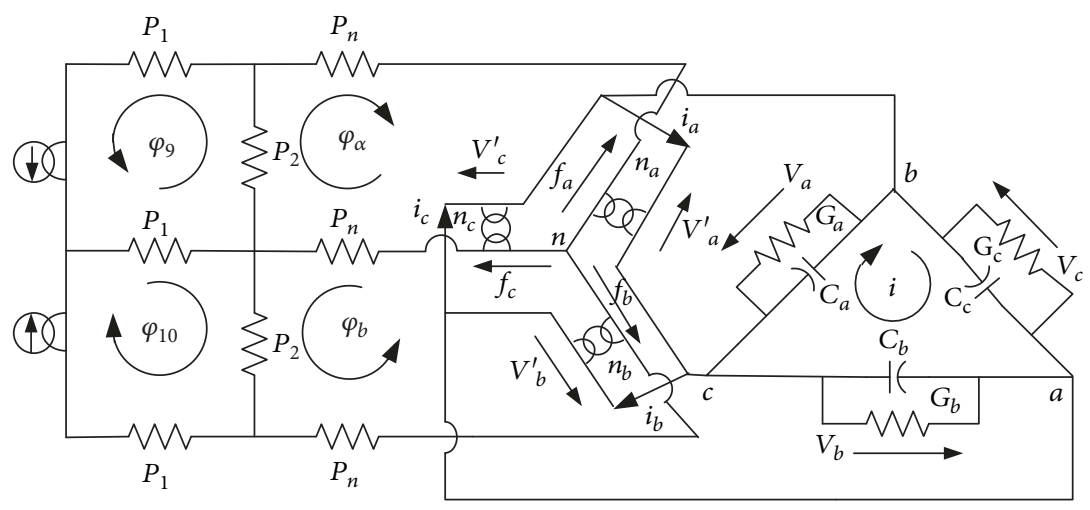

Figure 1: The equivalent circuit of the single- to three-phase converter circuit.

it demands nonlinear methods for analysis. Basically, two modeling strategies, namely, continuous-time averaging approach (CTAA) and discrete-time iterative map approach (DTIA), are used to formulate converter models. The CTAA is simple and more suitable to characterize low-frequency phenomena whereas DTIA provides detailed information on the dynamical behavior of the system. Investigation on these approaches reveals that the CTAA is suitable to study the behavior when regular periodic motion collapsed into a quasiperiodic orbit of a much longer period [8]. The interest on investigating switching converters increases since the system shows unusual bifurcation phenomena such as border collisions and grazings with usual properties of chaotic and quasiperiodic bifurcation.

Considerable works are identified on stabilizing such systems. Notable stabilization techniques that have been tested on electrical systems are time delayed approach $[9,10]$, artificial intelligence techniques, nonlinear feedback, sliding mode, and adaptive backstepping. In 2000, Chen et al. [11] studied the time-delayed feedback approach to stabilize the chaotic dynamics in an experimental DC drive. Asakura et al. [12] opted neural networks to stabilize the chaotic dynamics of an induction drive system. Nonlinear feedback control [13], sliding mode control [14], and adaptive backstepping control [15] have all been applied towards stabilization of chaos in electrical systems. After the contribution of Wang on the universal approximation theorem, the effective utilization of fuzzy logic is recognized in adaptive controllers [16]. A fuzzy logic-based adaptive controller is introduced in a MEMS triaxial gyroscope to approximate the model uncertainties and external disturbances [17]. Recently, Fang et al. [18] investigated vibration control of a cantilever beam with an adaptive backstepping fuzzy sliding mode controller and ascribed that the sliding mode control complements an additional compensator for achieving system stability. In order to reduce the influence of chattering effect, a nested dynamic sliding mode controller is formulated and robustness is proved for a three-phase active power filter [19]. An adaptive sliding mode control system using a double-loop recurrent neural network structure is designed and provided better approximation performance compared with the regular neural network approach [20]. In [21], it is shown that an adaptive sliding mode control with the fuzzy neural network based on a radial basis function can promise that the system could reach the sliding surface and converge to equilibrium point asymptotically.

Fractional calculus is a generalization of ordinary differentiation and integration to an arbitrary (noninteger) order. Exponential laws are a classical approach to study dynamical changes in systems, but there are many systems where dynamics undergo faster or slower changes than exponential laws. Fractional-order systems play a significant role to describe the irregular changes in dynamics. Naturally, dynamical systems are composed in the form of fractional order, for example, the voltage-current relation of a semi-infinite lossy transmission line [22] or diffusion of the heat through a semi-infinite solid, where heat flow is equal to the halfderivative of the temperature [23]. Recent studies reveal that fractional-order treatment allows us to describe and model a real object more accurately and precisely. Hartley et al. introduced the fractional-order Chua system with three single differential equations containing the noninteger (fractional) order derivatives [24]. The fractional term can generate a new degree of freedom which can significantly improve the control performance as well as the parameter identification [25].

Chaos suppression research findings on the permanent magnet synchronous generator (PMSG) in wind turbine [26], permanent magnet synchronous motor (PMSM) [27], brushless DC motor [28, 29], and smart grid [17] expose the unidentified chaotic regions while treating them as fractional orders. To the best of our knowledge, there is no power converter model with fractional order reported. In this paper, we formulated the fractional-order three-phase converter with saturable inductors from $[28,29]$.

\section{Phase Converter Circuit (PCC)}

In this paper, we consider the phase converter circuit with saturable inductors as described in $[28,29]$. The equivalent circuit of the single phase to the three-phase converter circuit of Ferro resonance type as given in [28] is shown in Figure 1.

The magneto motive force $f(\varphi)$ is related to the flux $\phi$ by a nonlinear relation $f(\varphi)=a_{1} \varphi+a_{3} \varphi^{2}$, and the other elements in the circuit shown in Figure 1 are assumed to be linear. The circuit equations are derived as magnetic and 
electrical subcircuits. The magnetic subcircuit equations can be derived as

$$
\begin{gathered}
f_{a}-f_{c}-f\left(\varphi_{c}\right)+f\left(\varphi_{a}\right)+P_{2}\left(\varphi_{a}+\varphi_{9}\right)=0, \\
f_{b}-f_{c}-f\left(\varphi_{c}\right)+f\left(\varphi_{b}\right)+P_{2}\left(\varphi_{b}+\varphi_{10}\right)=0,
\end{gathered}
$$

and the electrical subcircuit equations can be derived as

$$
\begin{gathered}
a \frac{d V_{a}}{d t}+G_{a} V_{a}+i_{c}=C_{c} \frac{d V_{c}}{d t}+G_{c} V_{c}+i_{a}, \\
C_{b} \frac{d V_{b}}{d t}+G_{b} V_{b}+i_{c}=C_{c} \frac{d V_{c}}{d t}+G_{c} V_{c}+i_{a} .
\end{gathered}
$$

We also assume that the variables are restricted to

$$
\begin{gathered}
\varphi_{a}+\varphi_{b}+\varphi_{c}=0, \\
V_{a}+V_{b}+V_{c}=0 .
\end{gathered}
$$

For simplicity, let us assume (symmetrical case) $C_{a}=C_{b}=C_{c}=C, G_{a}=G_{b}=G_{c}=G, N_{1}=N_{2}=N, \quad n_{a}=n_{b}$ $=n_{c}=n, \varphi_{9}=\varphi_{10}=\varphi(t)=\varphi_{m} \cos \omega t$, and $V_{a}=n\left(d \varphi_{a} / d t\right)$, $V_{b}=n\left(d \varphi_{b} / d t\right)$, and $V_{c}=n\left(d \phi_{c} / d t\right)$.

Using the relations discussed in (1), (2), and (3) and using the assumptions, the two state variables are taken as $x=\varphi_{a}-\varphi_{b}=\varphi, y=\varphi_{c}$ and $\tau=\omega t$; the state equations of Duffing type can be derived as

$$
\begin{aligned}
\ddot{x}+k \dot{x}+\left(c_{0}+c_{1}\right) x+\frac{c_{3}}{4}\left(x^{2}+3 y^{2}\right) x & =0, \\
\ddot{y}+k \dot{y}+\left(\frac{c_{0}}{3}+c_{1}\right) y+\frac{c_{3}}{4}\left(x^{2}+3 y^{2}\right) y & =B \cos \tau+B_{0},
\end{aligned}
$$

where $k=G / \omega C, c_{0}=P_{2} / \omega^{2} n^{2} C, c_{1}=a_{1} / \omega^{2} n^{2} C, c_{3}=a_{3} / \omega^{2} n^{2}$ $C, B=2 P_{2} \phi_{m} / 3 \omega^{2} n^{2} C$. To further derive the phase converter state equations, we use $c_{0}=c_{1}=0, c_{3}=1$ and $k=$ 0.1 as detailed in [28]. The value of $k$ is taken less than 0.3 to avoid nonperiod doubling bifurcations. Let $a=k$, $b=c_{3} / 4, c=3 c_{3} / 4, d=B, e=B_{0}$ and the state equations for the autonomous case can be derived as

$$
\begin{aligned}
& \dot{x}=y, \\
& \dot{y}=-a y-b x^{3}-c x z^{2}, \\
& \dot{z}=w, \\
& \dot{w}=-a w-b x^{2} z-c z^{3}+d \cos (u)+e, \\
& \dot{u}=1 .
\end{aligned}
$$

The PCC system described in (5) shows chaotic oscillations for the parameter values $a=0.1, b=0.25$, $c=0.75, \mathrm{~d}=3, e=0.2$ as discussed in $[28,29]$.

\section{Fractional-Order Phase Converter Circuit (FOPCC)}

This paper is aimed at analyzing the PCC system in its fractional-order form and also proposing sliding mode controllers to suppress chaotic oscillations with external disturbances and parameter uncertainties. To derive the fractional-order PCC (FOPCC), we have three commonly used definition of the fractional-order differential operator: Grünwald-Letnikov (GL), Riemann-Liouville, and Caputo [30-32]. Compared to the other two methods, GrünwaldLetnikov gives a smooth derivative because of the recursively defined binomial coefficients [33-37]. The continuous Riemann-Liouville approach is discretized using the GL method as seen in many literatures $[23,38,39]$ and involves a discrete convolution between a "weight" or binomial coefficient function and the function of interest for differentiation.

As can be seen in the literatures, the Caputo method has been widely used for numerical solutions of the fractionalorder systems. But the GL method has benefits over the other methods of solving fractional orders due to the smoothness of the resultant approximations [40]. Hence, we use the GL method to derive the FOPCC. The GL derivative can be defined as

$$
\begin{aligned}
{ }_{a} D_{t}^{q} f(t) & =\lim _{h \rightarrow 0}\left\{\frac{1}{h^{q}} \sum_{j=0}^{[t-a / h]}(-1)^{j}\left(\begin{array}{l}
q \\
j
\end{array}\right) f(t-j h)\right\} \\
& =\lim _{h \rightarrow 0}\left\{\frac{1}{h^{\alpha}} \Delta_{h}^{q} f(t)\right\} .
\end{aligned}
$$

For numerical calculations, the above equation is modified as

$$
(t-L) D_{t}^{q} f(t)=\lim _{h \rightarrow 0}\left\{h^{-q} \sum_{j=0}^{N(t)} \beta_{j}(f(t-j h))\right\}
$$

and the binomial coefficients required for the numerical simulation are calculated as

$$
\beta_{j}=\left(1-\frac{a+q}{j}\right) \beta_{j-1}
$$

Let the general form of the 3D fractional-order system be defined as

$$
\begin{aligned}
& D^{q_{x}}=A_{1}(x, y, z, w, u, t), \\
& D^{q_{y}}=A_{2}(x, y, z, w, u, t), \\
& D^{q_{z}}=A_{3}(x, y, z, w, u, t), \\
& D^{q_{w}}=A_{4}(x, y, z, w, u, t), \\
& D^{q_{u}}=A_{5}(x, y, z, w, u, t) .
\end{aligned}
$$

In order to simulate system (9) using the GL method, we use the discretization method discussed [40]: 


$$
\begin{aligned}
y\left(t_{k}\right)= & A_{2}\left(x\left(t_{k-1}\right), y\left(t_{k-1}\right), z\left(t_{k-1}\right), w\left(t_{k-1}\right), u\left(t_{k-1}\right)\right) h^{q_{y}} \\
& -\sum_{j=1}^{N} \beta_{j}^{q_{y}} y\left(t_{k-j}\right), \\
z\left(t_{k}\right)= & A_{3}\left(x\left(t_{k-1}\right), y\left(t_{k-1}\right), z\left(t_{k-1}\right), w\left(t_{k-1}\right), u\left(t_{k-1}\right)\right) h^{q_{z}} \\
& -\sum_{j=1}^{N} \beta_{j}^{q_{z}} z\left(t_{k-j}\right), \\
z\left(t_{k}\right)= & A_{3}\left(x\left(t_{k-1}\right), y\left(t_{k-1}\right), z\left(t_{k-1}\right), w\left(t_{k-1}\right), u\left(t_{k-1}\right)\right) h^{q_{z}} \\
& -\sum_{j=1}^{N} \beta_{j}^{q_{z}} z\left(t_{k-j}\right), \\
w\left(t_{k}\right)= & A_{4}\left(x\left(t_{k-1}\right), y\left(t_{k-1}\right), z\left(t_{k-1}\right), w\left(t_{k-1}\right), u\left(t_{k-1}\right)\right) h^{q_{w}} \\
& -\sum_{j=1}^{N} \beta_{j}^{q_{w}} w\left(t_{k-j}\right), \\
& -\sum_{j=1}^{N} \beta_{j}^{q_{u}} u\left(t_{k-j}\right),
\end{aligned}
$$

where $\beta$ is the binomial coefficient calculated using (8). The value of $N$ is taken as the truncation window size and $L$ is the memory element available and $k$ when all the available memory elements are used.

Let us define the FOPCC oscillator as

$$
\begin{aligned}
D^{q_{x}} x & =y, \\
D^{q_{y}} y & =-a y-b x^{3}-c x z^{2}, \\
D^{q_{z}} z & =w, \\
D^{q_{w}} w & =-a w-b x^{2} z-c z^{3}+d \cos (u)+e, \\
D^{q_{u}} u & =1 .
\end{aligned}
$$

Using (10) in (11), the discrete form of the FOPCC system can be derived as

$$
\begin{aligned}
x\left(t_{k}\right)= & \left(y\left(t_{k-1}\right)\right) h^{q_{x}}-\sum_{j=1}^{N} \beta_{j}^{q_{x}} x\left(t_{k-j}\right), \\
y\left(t_{k}\right)= & \left(-a y\left(t_{k-1}\right)-b x\left(t_{k-1}\right)^{3}-c x\left(t_{k-1}\right) z\left(t_{k-1}\right)^{2}\right) h^{q_{y}} \\
& -\sum_{j=1}^{N} \beta_{j}^{q_{y}} y\left(t_{k-j}\right), \\
z\left(t_{k}\right)= & \left(w\left(t_{k-1}\right)\right) h^{q_{z}}-\sum_{j=1}^{N} \beta_{j}^{q_{z}} z\left(t_{k-j}\right), \\
w\left(t_{k}\right)= & \left(-a w\left(t_{k-1}\right)-b x\left(t_{k-1}\right)^{2} z\left(t_{k-1}\right)-c z\left(t_{k-1}\right)^{3}\right. \\
& \left.+d \cos \left(u\left(t_{k-1}\right)\right)+e\right), \\
u\left(t_{k}\right)= & (1) h^{q_{u}}-\sum_{j=1}^{N} \beta_{j}^{q_{u}} u\left(t_{k-j}\right) .
\end{aligned}
$$

For the parameter values $a=0.1, b=0.25, c=0.75$, $e=0.2$, fractional order $q=0.995$, and initial conditions $[1,1,1,1,1]$, the FOPCC system (11) $2 \mathrm{D}$ phase portrait is shown in Figure 2 and chaotic oscillations also shown in Figure 2. In the entire numerical analysis of the FOPCC, the step size is $h=0.001$.

\section{Dynamical Properties of the FOPCC System}

4.1. Bifurcation. We discuss the bifurcation of the FOPCC with respect to parameters and fractional order. The autonomous FOPCC system shown in (5) has five parameters. Our interest falls on parameter $d$ for the reason that it represents the amplitude of the external excitation of the Duffing type equation. In [28, 29], numerical bifurcation analysis was done, but system behavior for various values of the parameters is not sufficiently analyzed. Even though numerical bifurcation analysis was partly done in $[28,29]$, fewer have been investigated on the system behavior for various values of the parameters. We hence derive the bifurcation plots with the other parameters taken as $a=0.1, b=0.25, c=0.75$, $e=0.2$, fractional order $q=0.995$, and using $d$ as the control parameter and the initial conditions taken as $[1,1,1,1,1]$. Figure 3 shows the bifurcation of the FOPCC with $d$. As can be seen from Figure 3, the FOPCC system shows period-1 limit cycles for $2 \leq d \leq 2.6$, and at $d=2.6$, the first period doubling is seen. For region $2.694 \leq d \leq$ 3.988 , the FOPCC system shows the first chaotic zone and exits chaos and shows period-3 limit cycles for $3.989 \leq d \leq$ 7.694. We could see the second chaotic region for $7.8 \leq d \leq$ 8 , and the FOPCC system exits chaos and shows period- 3 oscillations for $8.001 \leq d \leq 8.756$ and period- 6 oscillations for $8.757 \leq d \leq 8.932$. The third chaotic region of the system is seen for $8.98 \leq d \leq 9.56$ and exits chaos with period-2 limit cycles.

Figure 3(b) shows the bifurcation of the FOPCC system with the fractional order $q$ which is varied between $[0.98,1]$ while keeping the other parameters as $a=0.1, b=0.25$, $c=0.75, \mathrm{~d}=3, e=0.2$ and the initial conditions taken as $[1,1,1,1,1]$. The FOPCC shows chaotic oscillations for $q>0.989$, and we could see transient spirals in the region $0.98 \leq q \leq 0.986$ which is mainly due to the effect of the truncation in the calculation of binomial coefficients. To remove such spiral transients, we could select a larger memory size which would result in longer simulation times.

4.2. Multistability and Coexisting Attractors. Multistability and coexisting attractors in physical systems play a significant role as such oscillations may create hazardous effects in system behavior. There are few discussions on the multistable properties of mechanical systems such as the controllability of multistability in a quasiperiodically driven system [41-43] and multistability and coexisting attractors in a horizontal platform system [44]. But there are no such investigations of coexisting attractors in an electrical system and especially in fractional-order physical systems. Hence, we are interested in investigating the multistability feature of the FOPCC system. 

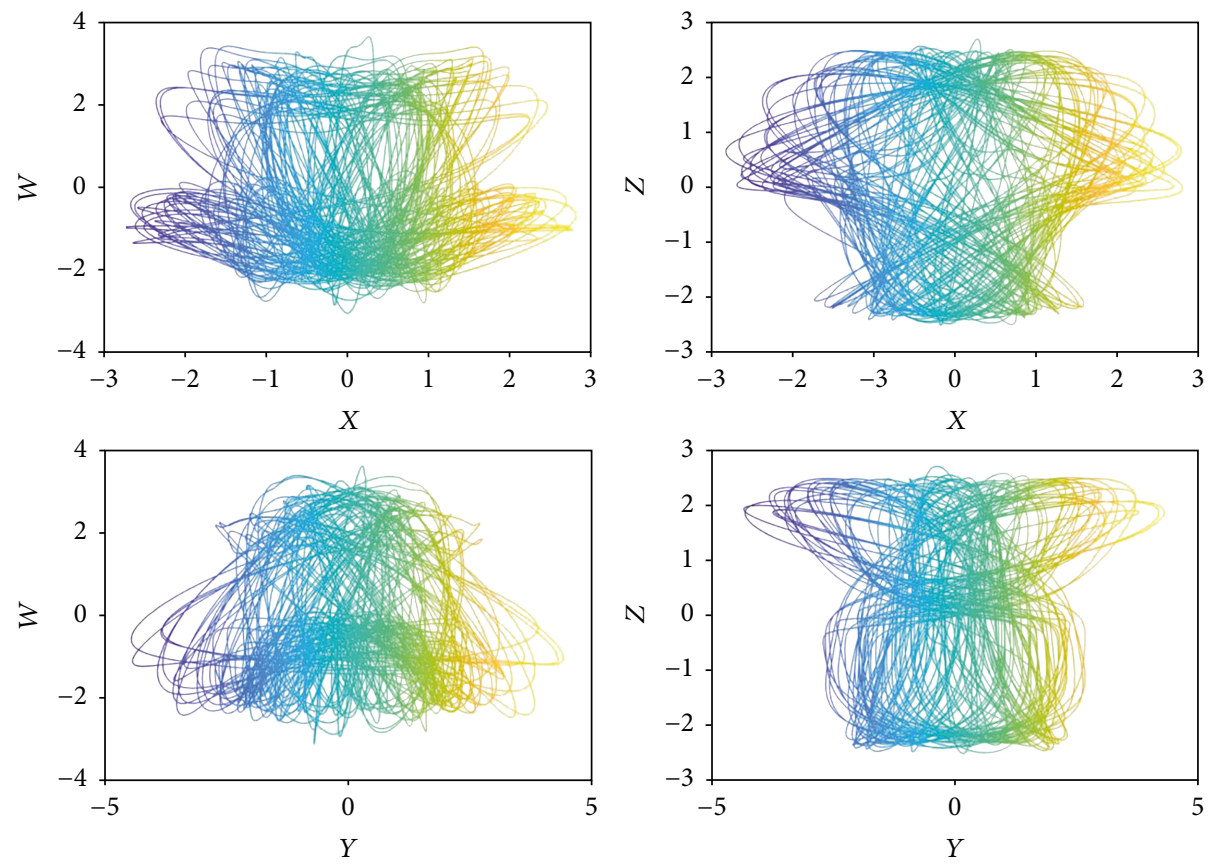

(a)
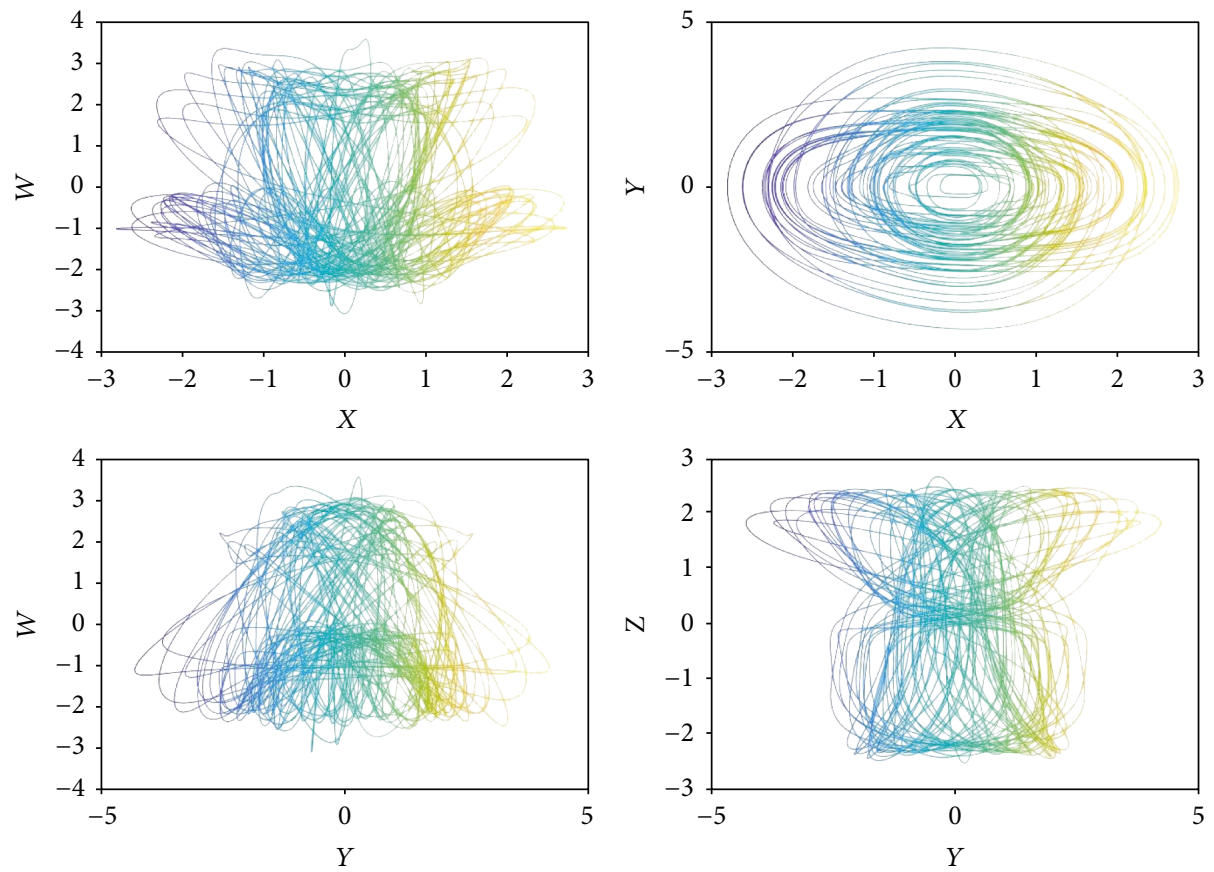

(b)

Figure 2: 2D phase plots of the FOPCC system for (a) $d=3$ and (b) $d=9.5$ with the initial conditions $[1,1,1,1,1]$.

To derive the multistability plots of the FOPCC, the bifurcation diagram is obtained by plotting local maxima of the coordinate $x$ in terms of the parameter that is increased (or decreased) in tiny steps in the range of $2 \leq d \leq 10$ with the parameters $a=0.1, b=0.25, c=0.75, e=0.2$ and the fractional order $q=0.995$. The initial condition for the first iteration is taken as $[1,1,1,1,1]$ and is reinitialized to the end values of the state variables in every iteration. This strategy, known as forward and backward continuation, represents a simple way to localize the window in which the system develops multistability. The existence of multistability can be confirmed by comparing the forward (Figure 4(a) blue) and backward (Figure 4(a) red) bifurcation diagrams. As can be seen from Figure 4(a), a chaotic attractor coexists with a period-2 limit cycle in the regions $7.8 \leq d \leq 8,8.001 \leq d \leq$ 8.756 , and $9.89 \leq d \leq 10$. The corresponding Lyapunov exponents (LEs) for forward (Figure 4(b)) and backward (Figure 4(c)) are also presented to confirm the findings on coexisting attractors. The LEs are calculated using the wellknown Wolfs algorithm [45] by replacing the ODE integrator 


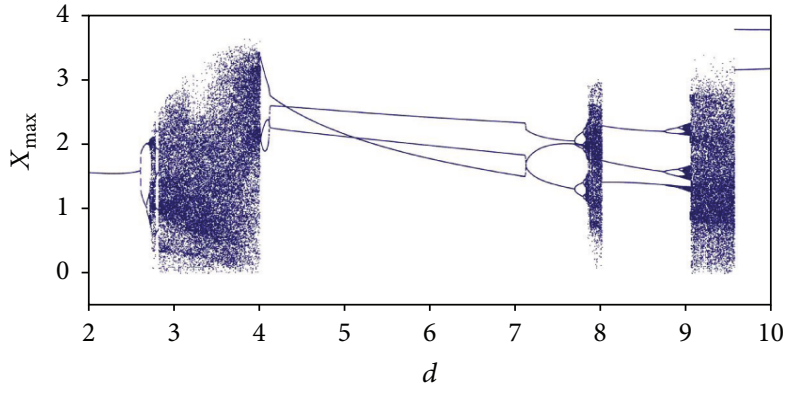

(a)

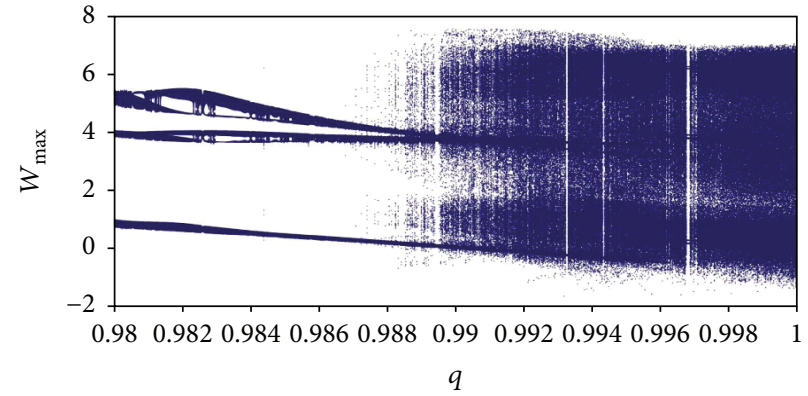

(b)

Figure 3: (a, b) The bifurcation of the FOPCC system with $d$.

for continuous flow with fde12 $[26,27]$ which is based on the Adam-Bashforth-Moulton predictor corrector method. Figures 5(a)-5(c) shows the coexisting attractors of the FOPCC system.

\section{Chaos Suppression in the Phase Converter Circuit}

Recent literatures have shown that fractional-order controls are effective in suppressing chaos compared to their integer-order counterparts [1-8, 22, 24]. Engineering systems which change under conditions demanding closed loops, various control schemes were used to get desirable output. PID controllers are a widely used control algorithm in general engineering applications. But systems with sensitivity in initial conditions and with drastic changes in parameters such as chaotic and hyperchaotic systems need an advanced control algorithm. An adaptive control scheme is introduced and shows effectiveness in suppressing chaos $[1,6]$. The extended backstepping control algorithm provided a successful output in [2]. Three famous control schemes named as sliding mode control, robust control, and extended backstepping control are implemented in fractional-order brushless DC motor to suppress chaos [4]. The sliding mode control is free from chattering effects, so it is more suitable for control of complex nonlinear systems like the fractional-order Willamowski-Rössler chemical system [5]. For systems suffering from external disturbances and/or parameter uncertainties, the sliding mode controller adapts to the sliding condition first so the adaptive sliding mode control algorithm is introduced [7]. Nonlinear phenomena in complex systems such as voltage collapse and oscillatory phenomenon in power systems can be studied using the chaos theory. Chaos control methodologies have been of greater interest in nonlinearity suppression of power systems. Chaos control problems in fractional-order complex systems with higher harmonics like the induction motor drive system and smart grid systems are discussed where the control objective is achieved with a genetically optimized fractional-order PID controller [3, 8].

We discuss the chaos suppression problem of FOPCC with two different scenarios. In the first one, we assume that the FOPCC system has model uncertainties and external disturbances and design a fractional-order no-chattering sliding mode controller to suppress chaotic oscillations. In the second scenario, we assume that the FOPCC system has parameter uncertainties and design a fractional-order adaptive sliding mode controller to suppress chaos. In both cases, we use the Lyapunov approach to show that the designed controllers are effective in suppressing chaos in finite time $t$.

5.1. Chaos Suppression of the FOPCC System with Disturbances. In this section, we derive the chaos control for the FOPCC system considered with external disturbances. We use the no-chattering sliding mode control defined in [24]. Let us define the nonautonomous FOPCC system with the controllers as

$$
\begin{aligned}
D^{q_{x}} x & =y+u_{1}, \\
D^{q_{y}} y & =-a y-b x^{3}-c x z^{2}+u_{2}, \\
D^{q_{z}} z & =w+u_{3}, \\
D^{q_{w}} w & =-a w-b x^{2} z-c z^{3}+d \cos (t)+e+u_{4} .
\end{aligned}
$$

Let the nonlinear generalized equation of the FOPCC system (13) be defined as

$$
\dot{x}=A x+C b+C u+d+\Delta p,
$$

where $A$ is the system matrix, $C$ is the control matrix, $d$ is the disturbance, $b$ is the nonlinearity in the FOPCC system, and $\Delta p$ is the parameter. Let us assume that the disturbances and the parameters are bounded to $|d| \leq \alpha_{1}$ and $|\Delta p| \leq \alpha_{2}$, respectively. The desired signal for control can be defined as $x_{d}=\left[x_{d_{x}} x_{d_{y}} x_{d_{z}} x_{d_{w}}\right]$.

The error dynamics of the system defined in (14) is

$$
D^{q} e=D^{q} x-D^{q} x_{d}=A x+C b+C u+d+\Delta p-D^{q} x_{d} .
$$

The proportional integral sliding surface can be defined as

$$
S=\lambda e-\int_{0}^{t} \lambda(A-C L) e(\tau) d \tau
$$




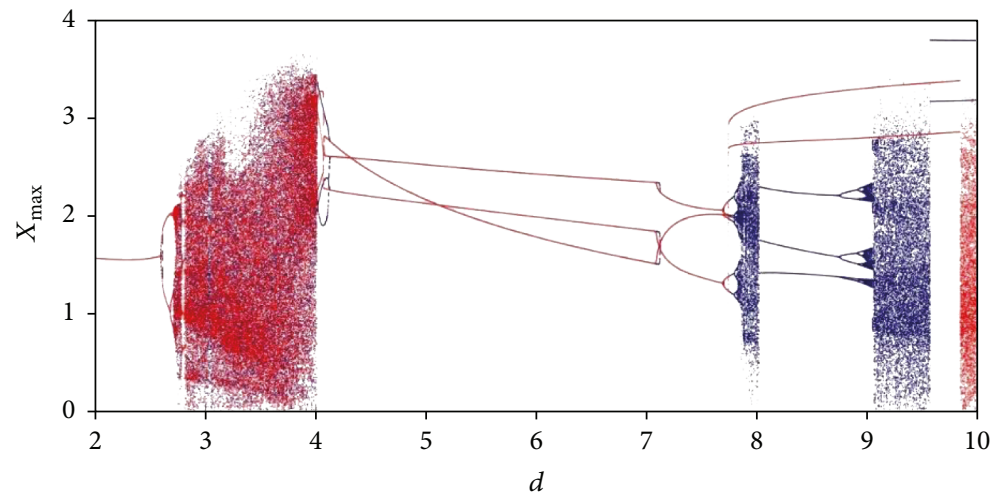

(a)
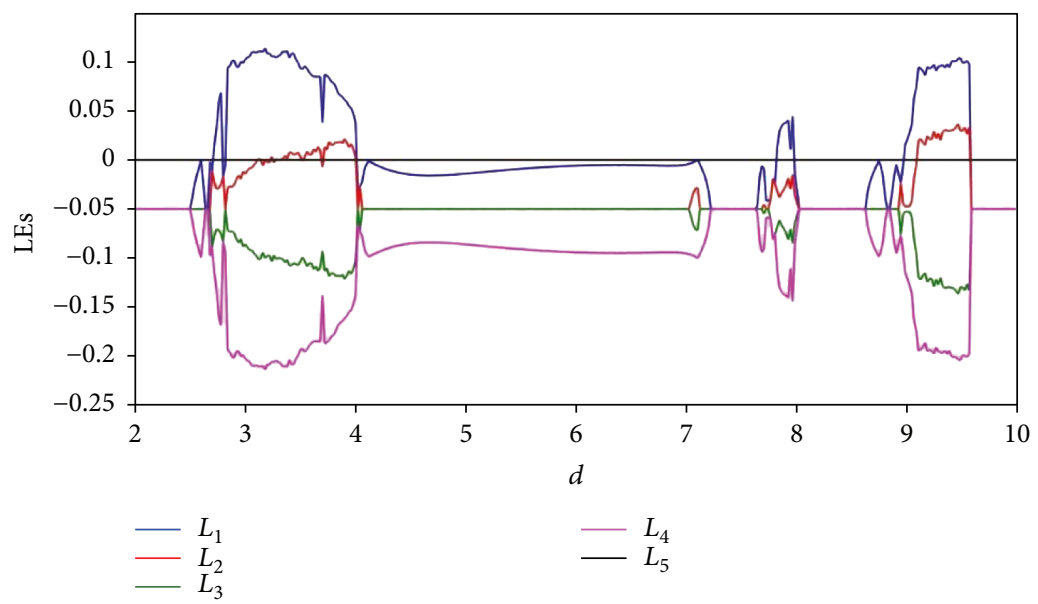

(b)

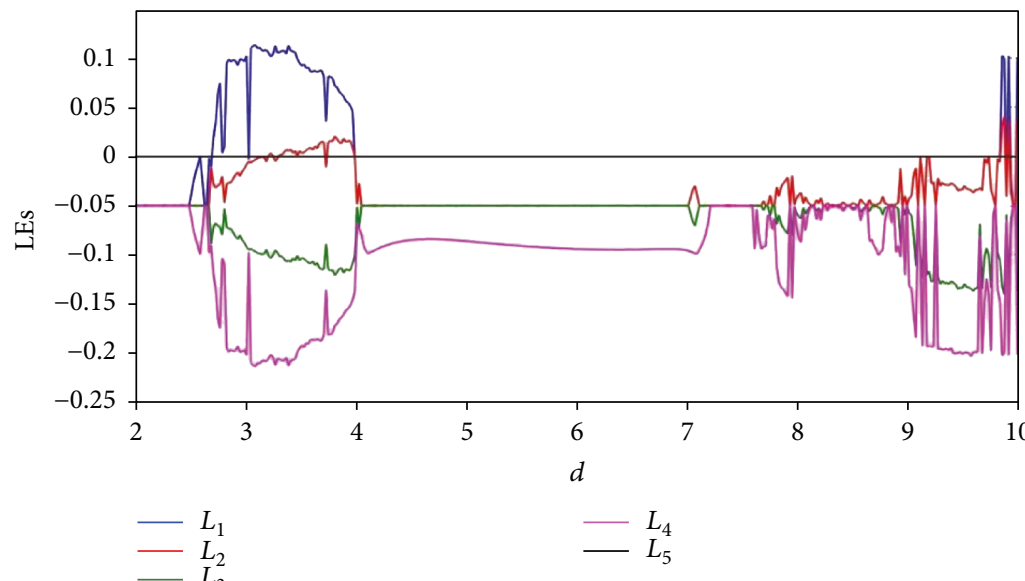

(c)

FIgURE 4: (a) Bifurcation plots of the FOPCC system with parameter $d$ where the forward continuation is shown with blue dots and backward continuation is shown with red dots. The corresponding LEs are shown in (b) and (c) for forward and backward continuation, respectively.

where $\lambda \in R^{4 \times 4}$ such that $\operatorname{det}(\lambda C) \neq 0$ and $L \in R^{4 \times 4}$ such that the eigenvalues of the matrix $|A-C L|$ are negative and hence stable.

In the sliding surface, we know the condition that $S=D^{q} S=0$ and hence

$$
D^{q} S=\lambda D^{q} e-\lambda(A-C L) e .
$$

Using (14) and (15) in (16),

$$
D^{q} S=C \lambda b+C \lambda u+\lambda d+\lambda \Delta p+A \lambda x_{d}-\lambda D^{q} x_{d}+C L \lambda e .
$$

The controller satisfying the sliding condition can be defined as 


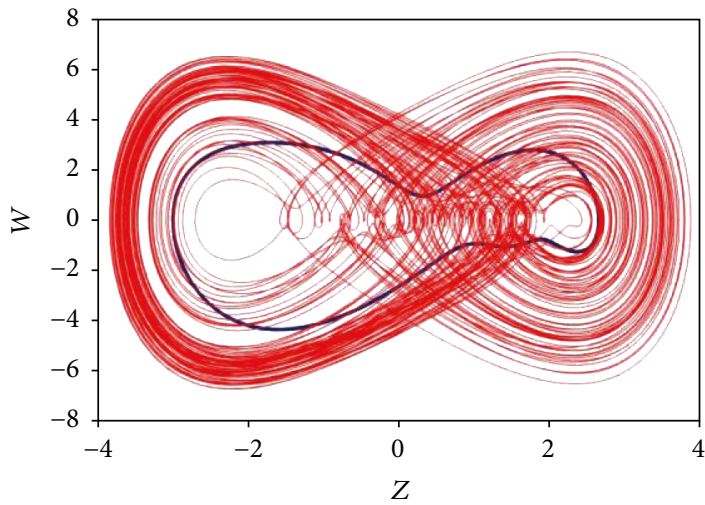

(a)

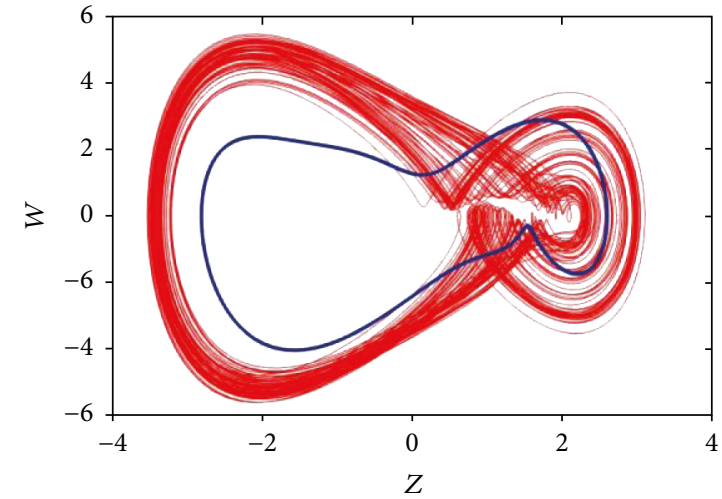

(b)

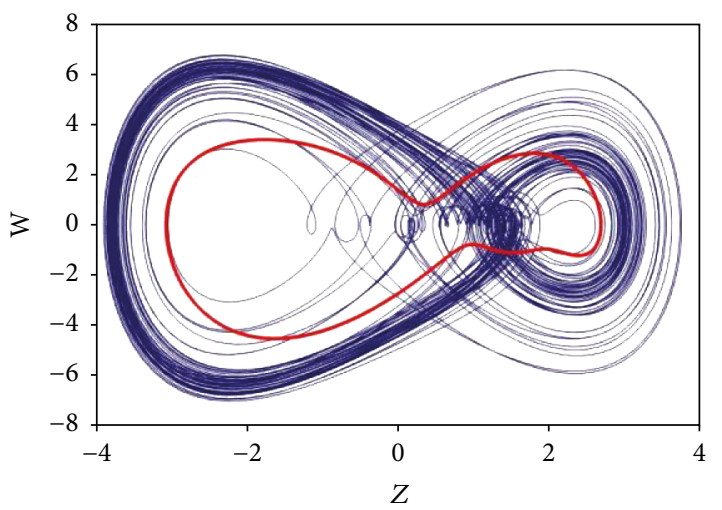

(c)

Figure 5: Coexisting attractors exhibited by the FOPCC system. (a) Coexisting attractor and period-1 limit cycle for $d=9.5$ with initial conditions $[-3.59,-2.73,-1.34,2.86,1]$ shown as blue plot and with initial conditions $[1,1,1,1,1]$ shown as red plot. (b) Coexisting attractor and period- 1 limit cycle for $d=8$ with initial conditions $[1.53,-5.49,2.39,-1.74,1]$ shown as blue plot and with initial conditions $[1,1,1,1,1]$ shown as red plot. (c) Coexisting attractor and period-1 limit cycle for $d=10$ with initial conditions $[1.53,-5.49,2.39,-1.74,1]$ shown as blue plot and with initial conditions $[1,1,1,1,1]$ shown as red plot.

$$
\begin{aligned}
u= & -(b+L e)-(C \lambda)^{-1}\left[A \lambda x_{d}-\lambda D^{q} \dot{x}_{d}\right] \\
& -(C \lambda)^{-1}[\mu+\|C \lambda b\|] G\left(\frac{S}{h}\right),
\end{aligned}
$$

where

$$
G\left(\frac{S}{h}\right)=\left\{\begin{array}{cc}
\operatorname{sgn}(S) & |S|>h \\
\frac{S}{h} & |S| \leq h
\end{array}\right\},
$$

and $h$ is a positive constant.

5.1.1. Stability of the Controller. We use the Lyapunov approach to derive the stability of the controller and to show that the controller can make the FOPCC system reach the sliding condition. Let us define the Lyapunov function as

$$
V=S_{1}^{2}+S_{2}^{2}+S_{3}^{2}+S_{4}^{2}=S^{T} \dot{S}
$$

The Lyapunov first derivative can be defined as

$$
\dot{V}=S^{T} D^{1-q} D^{q} S .
$$

The direct numerical solution of (22) is more complex, and hence, we use the modified Lyapunov method for the fractional order used in [4] to simplify (23) as

$$
\dot{V}=S^{T} D^{q} S .
$$

Using (18) in (23), we get

$\dot{V}=S^{T}\left[C \lambda b+C \lambda u+\lambda d+\lambda \Delta p+A \lambda x_{d}-\lambda D^{q} x_{d}+C L \lambda e\right]$.

Using the designed sliding mode controller (19) in (24),

$$
\dot{V}=S^{T}\left[\lambda d+\lambda \Delta p-(\mu+\|C L b\|) G\left(\frac{S}{h}\right)\right] .
$$

Further approximating (25), we can derive (26) as

$$
\dot{V} \leq S^{T}\left[d+\Delta p-\mu G\left(\frac{S}{h}\right)\right] .
$$


As per the boundedness of the disturbance and the parameters,

$$
\dot{V} \leq \sum_{i=1}^{4}\left|S_{i}\right| \alpha_{1}+\sum_{i=1}^{4}\left|S_{i}\right| \alpha_{2}-\sum_{i=1}^{4} S_{i} G\left(\frac{S}{h}\right) \mu .
$$

If we assume $|S|>h$, then $S_{i} G\left(S_{i} / h\right)=\left|S_{i}\right|$ and (27) modify to

$$
\dot{V} \leq \sum_{i=1}^{4}\left|S_{i}\right|\left[\alpha_{1}+\alpha_{2}-\mu\right]
$$

If the positive constant $\mu>\alpha_{1}+\alpha_{2}+\beta$ and with $\beta>0$, the controller (19) will make system (13) reach the sliding condition.

$$
\dot{V} \leq \beta \sum_{i=1}^{4}\left|S_{i}\right|
$$

The Lyapunov derivative is negative semidefinite, confirming that there exists a finite time $T$ where $|S| \leq h$.

\subsubsection{Numerical Analysis. For the FOPCC system,}

$$
\begin{aligned}
& A=\left[\begin{array}{cccc}
0 & 1 & 0 & 0 \\
0 & -a & 0 & 0 \\
0 & 0 & 0 & 1 \\
0 & 0 & 0 & -a
\end{array}\right], \\
& C=\left[\begin{array}{llll}
1 & 0 & 0 & 0 \\
0 & 1 & 0 & 0 \\
0 & 0 & 1 & 0 \\
0 & 0 & 0 & 1
\end{array}\right] \text {, } \\
& d=\left[\begin{array}{l}
d_{1} \\
d_{2} \\
d_{3} \\
d_{4}
\end{array}\right] \\
& b=\left[\begin{array}{c}
0 \\
-b x^{3}-c x z^{2} \\
0 \\
-b x^{2} z-c z^{3}+d \cos (t)
\end{array}\right] \text {, } \\
& \Delta p=\left[\begin{array}{l}
0 \\
0 \\
0 \\
e
\end{array}\right] \text {. }
\end{aligned}
$$

For numerical simulations, the initial conditions of the nonautonomous FOPCC system are taken as $[1,1,1,1]$ and the disturbances are $[0.8 \sin (0.5 t) ; 1.2 \sin (0.4 t) ; 0.4 \sin$ $(t) ; \sin (2 t)]$. The gain matrix is taken as $\lambda=\operatorname{diag}(1,1,1,1)$, and the eigenvalues of $A-C L$ are assumed to be $[-3,-3$, $-3,-3]$ and hence

$$
L=\left[\begin{array}{cccc}
3 & 1 & 0 & 0 \\
0 & 3-a & 0 & 0 \\
0 & 0 & 3 & 1 \\
0 & 0 & 0 & 3-a
\end{array}\right]
$$

For simplicity, the desired signal is taken as $[0.5 \mathrm{sin}$ $(t), 1.2 \sin (5 t), 1.5 \sin (2 t), 0.2 \sin (1.5 t)]$. Figure 6 shows the time history of the controlled states with the controllers switched on at $t=20 \mathrm{~s}$.

5.2. Chaos Suppression of FOPCC System with Parameter Uncertainties. In this section, we assume that the FOPCC system has uncertain parameters and hence we derive an adaptive sliding mode controller which could estimate the parameters and suppress chaotic oscillations. Let us define the FOPCC system with adaptive sliding mode controllers as

$$
\begin{aligned}
& D^{q} x=y, \\
& D^{q} y=-a y-b x^{3}-c x z^{2}+u_{1}, \\
& D^{q} z=\omega, \\
& D^{q} \omega=-a \omega-b x^{2} z-c z^{3}+d \cos (t)+e+u_{2} .
\end{aligned}
$$

The uncertainties in the parameters are estimated with the parameter estimates $\widehat{a}, \widehat{b}, \widehat{c}, \widehat{d}, \widehat{e}$, and the parameter estimation errors are defined as

$$
e_{i}=\widehat{i}-i \quad \text { for } i \in\{a, b, c, d, e\} \text {. }
$$

The dynamics of the parameter estimates are derived as

$$
D^{q_{i}} e_{i}=D^{q_{i}} \widehat{i} \quad \text { for } i \in\{a, b, c, d, e\} .
$$

Let us define the proportional integral sliding surface as

$$
\begin{gathered}
s_{y}=y+\lambda_{y} \int y d \tau, \\
s_{w}=w+\lambda_{w} \int w d \tau .
\end{gathered}
$$

The fractional-order sliding dynamics can be derived from (35):

$$
\begin{aligned}
D^{q_{y}} s_{y}= & D^{q_{y}} y+\lambda_{y} y=-a y-b x^{3}-c x z^{2}+u_{y}+\lambda_{y} y, \\
D^{q_{w}} s_{w}= & D^{q_{w}} w+\lambda_{w} w=-a w-b x^{2} z-c z^{3}+d \cos (t) \\
& +e+u_{w}+\lambda_{w} w .
\end{aligned}
$$




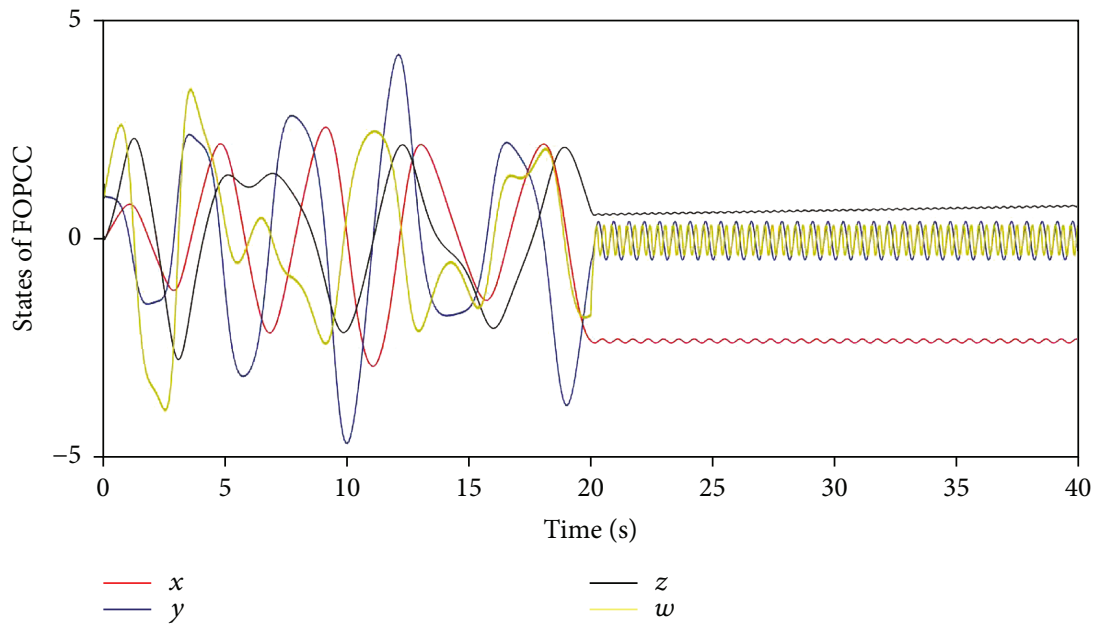

FIGURE 6: The time history of the states with the controller in effect when $t \geq 20 \mathrm{~s}$ and the initial conditions for the simulation is $[0,1,0,1]$, and the commensurate fractional order is $q=0.995$.

Let us define the adaptive controllers which can make the FOPCC system with parameter uncertainties reach the sliding condition as

$$
\begin{aligned}
u_{y}= & \widehat{a} y+\widehat{b} x^{3}+\widehat{c} x z^{2}-\lambda_{y} y-\eta_{y} \operatorname{sgn}\left(s_{y}\right)-\rho_{y} s_{y}, \\
u_{w}= & \widehat{a} w+\widehat{b} x^{2} z+\widehat{c} z^{3}-\widehat{d} \cos (t)-\widehat{e}-\lambda_{w} w \\
& -\eta_{w} \operatorname{sgn}\left(s_{w}\right)-\rho_{w} s_{w}
\end{aligned}
$$

where $\eta_{y}, \eta_{w}, \rho_{y}, \rho_{w}, \lambda_{y}, \lambda_{w}$ are all positive constants.

5.2.1. Stability of the Controllers. The stability of the controllers is established using the Lyapunov stability approach. The Lyapunov candidate function can be defined as

$$
V=\frac{1}{2} s_{y}^{2}+\frac{1}{2} s_{w}^{2}+\frac{1}{2} e_{i}^{2} \quad \text { for } i \in\{a, b, c, d, e\}
$$

The dynamics of the Lyapunov candidate function is

$$
\dot{V}=s_{y} \dot{s}_{y}+s_{w} \dot{s}_{w}+e_{i} \dot{e}_{i} .
$$

From the definition of fractional calculus,

$$
\dot{V}=s_{y} D^{q_{y}} D^{1-q_{y}} s_{y}+s_{w} D^{q_{w}} D^{1-q_{w}} s_{w}+e_{i} D^{q_{i}} D^{1-q_{i}} e_{i} .
$$

The direct numerical simulation of (41) is complex, and hence, we use the method as that used to solve (22) and (41) can be simplified as

$$
\begin{aligned}
\dot{V}= & \left\{s_{y}\left[-a y-b x^{3}-c x z^{2}+u_{y}+\lambda_{y} y\right]\right. \\
& +s_{w}\left[-a w-b x^{2} z-c z^{3}+d \cos (t)+e+u_{w}+\lambda_{w} \mathrm{w}\right] \\
& \left.+e_{a} D^{q} \widehat{a}+e_{b} D^{q} \widehat{b}+e_{c} D^{q} \widehat{c}+e_{d} D^{q} \widehat{d}+e_{e} D^{q} \widehat{e}\right\},
\end{aligned}
$$

where $q$ is the commensurate fractional order of the system.
Using the controller (37) in (42),

$$
\begin{aligned}
\dot{V}= & \left\{s_{y}\left[e_{a} y+e_{b} x^{3}+e_{c} x z^{2}-\eta_{y} \operatorname{sgn}\left(s_{y}\right)-\rho_{y} s_{y}\right]\right. \\
& +s_{w}\left[e_{1} w+e_{b} x^{2} z+e_{c} z^{3}-e_{d} \cos (t)-e_{e}-\eta_{w} \operatorname{sgn}\left(s_{w}\right)\right. \\
& \left.\left.-\rho_{w} s_{w}\right]+e_{a} \dot{\vec{a}}+e_{b} \dot{\hat{b}}+e_{c} \dot{\vec{c}}+e_{d} \dot{\hat{d}}+e_{e} \dot{\hat{e}}\right\} .
\end{aligned}
$$

Let us define the parameter update laws as

$$
\begin{aligned}
& D^{q} \widehat{a}=-y s_{y}-w s_{w}, \\
& D^{q} \widehat{b}=-x^{3} s_{y}-x^{2} z s_{w}, \\
& D^{q} \widehat{c}=-x^{2} z s_{y}-z^{3} s_{w}, \\
& D^{q} \widehat{d}=\cos (t) s_{w}, \\
& D^{q} \widehat{d}=s_{w} .
\end{aligned}
$$

Using the Parameter update laws in (43),

$$
\dot{V}=-\eta_{y}\left|s_{y}\right|-\rho_{y} s_{y}^{2}-\eta_{w}\left|s_{w}\right|-\rho_{w} s_{w}^{2} .
$$

The Lyapunov first derivative in (45) is negative semidefinite confirming that the controller will make the FOPCC system reach the sliding condition in a finite time.

For numerical simulations, we use the initial conditions of the state variables as $[0,1,0,1]$ and the parameter estimates as $\widehat{a}=0, \widehat{b}=0, \widehat{c}=0, \widehat{d}=0, \widehat{e}=0$. The commensurate fractional order is $q=0.995$. Figures 7 and 8 show the time history of the parameter estimates and controlled states of the FOPCC system, respectively.

\section{Conclusion}

A fractional-order phase converter circuit is derived from its integer-order mathematical model, and various 


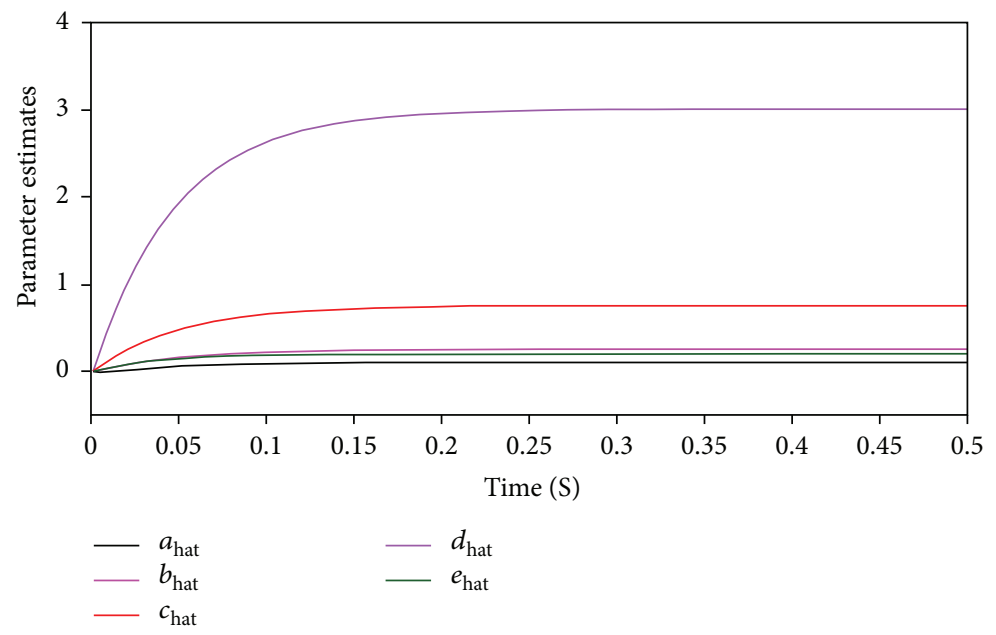

Figure 7: The time history of the parameter estimates of the FOPCC system.

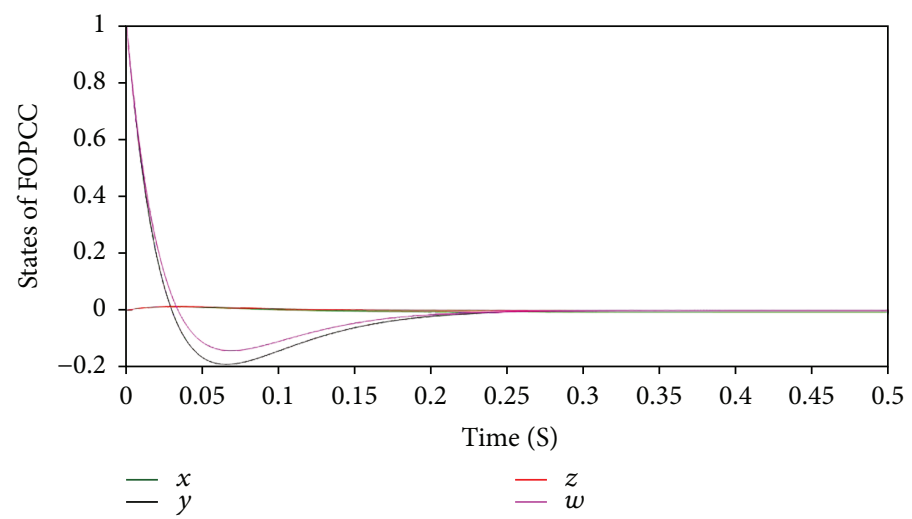

FIGURE 8: The time history of the controlled states of the FOPCC system.

dynamical properties are discussed. Bifurcation analysis of the fractional-order system with forward and backward continuation shows the existence of multistability. Such coexisting attractors in a power system model were not explored earlier, and multistability existence is hazardous in any physical nonlinear system and needs to be further investigated. Coexisting attractors shown by the fractional-order system are shown but not limited to only those discussed as there may be other initial conditions and parameters that can contribute to coexistence. To suppress chaotic oscillations, two different scenarios are discussed. In the first scenario, we use a chattering free sliding mode controller to suppress chaotic oscillations with disturbances, and in the second scenario, we use an adaptive sliding mode controller to control chaos with parameter uncertainties. Numerical simulations are conducted to validate the effectiveness of the controllers.

\section{Data Availability}

All the numerical simulation parameters are mentioned in the respective text part, and there are no additional data requirements for the simulation results.

\section{Conflicts of Interest}

The authors declare that there is no conflict of interest in publishing the paper.

\section{References}

[1] A. Karthikeyan, K. Rajagopal, and D. Mathew, "Fractional order nonlinear variable speed and current regulation of a permanent magnet synchronous generator wind turbine system," Alexandria Engineering Journal, vol. 57, no. 1, pp. 159-167, 2018.

[2] K. Rajagopal, A. Karthikeyan, and P. Duraisamy, "Chaos suppression in fractional order permanent magnet synchronous motor and PI controlled induction motor by extended back stepping control," Nonlinear Engineering, vol. 5, no. 4, 2016.

[3] A. Karthikeyan and K. Rajagopal, "Chaos control in fractional order smart grid with adaptive sliding mode control and genetically optimized PID control and its FPGA implementation," Complexity, vol. 2017, Article ID 3815146, 18 pages, 2017.

[4] K. Rajagopal, S. Vaidyanathan, A. Karthikeyan, and P. Duraisamy, "Dynamic analysis and chaos suppression in a fractional order brushless DC motor," Electrical Engineering, vol. 99, no. 2, pp. 721-733, 2017. 
[5] K. Rajagopal and A. Karthikeyan, "Chaos suppression of fractional order Willamowski-Rössler chemical system and its synchronization using sliding mode control," Nonlinear Engineering, vol. 5, no. 3, 2016.

[6] K. Rajagopal and A. Karthikeyan, "Chaos suppression for a fourth order memristor chaotic oscillator with uncertain parameters," International Journal of Control Theory and Applications, vol. 9, no. 38, pp. 751-762, 2016.

[7] K. Rajagopal, A. Karthikeyan, and P. Duraisamy, "Bifurcation analysis and chaos control of a fractional order portal frame with nonideal loading using adaptive sliding mode control," Shock and Vibration, vol. 2017, Article ID 2321060, 14 pages, 2017.

[8] K. Rajagopal, G. Laarem, A. Karthikeyan, and A. Srinivasan, "FPGA implementation of adaptive sliding mode control and genetically optimized PID control for fractional-order induction motor system with uncertain load," Advances in Difference Equations, vol. 2017, no. 1, 2017.

[9] K. Pyragas, "Continuous control of chaos by self-controlling feedback," Physics Letters A, vol. 170, no. 6, pp. 421-428, 1992.

[10] K. Pyragas, "Control of chaos via an unstable delayed feedback controller," Physical Review Letters, vol. 86, no. 11, pp. 22652268, 2001.

[11] J. H. Chen, K. T. Chau, S. M. Siu, and C. C. Chan, "Experimental stabilization of chaos in a voltage-mode DC drive system," IEEE Transactions on Circuits and Systems I: Fundamental Theory and Applications, vol. 47, no. 7, pp. 10931095, 2000.

[12] T. Asakura, K. Yoneda, Y. Saito, and M. Shioya, "Chaos detection in velocity control of induction motor and its control by using neural network," in WCC 2000 - ICSP 2000. 5th International Conference on Signal Processing Proceedings. 16th World Computer Congress 2000, vol. 3, pp. 1633-1638, Beijing, China, August 2000.

[13] H. Ren and D. Liu, "Nonlinear feedback control of chaos in permanent magnet synchronous motor," IEEE Transactions on Circuits and Systems II: Express Briefs, vol. 53, no. 1, pp. 45-50, 2006.

[14] W. Cheng, Y. Tong, and C. Li, "Chaos control of permanent magnet synchronous motor via sliding mode variable structure scheme," in 2011 3rd International Workshop on Intelligent Systems and Applications, pp. 1-4, Wuhan, China, May 2011.

[15] G. Xiaohui and H. Jin, "Chaos control of permanent magnet synchronous motor," in 2005 International Conference on Electrical Machines and Systems, vol. 1, pp. 484-488, Nanjing, China, September 2005.

[16] L. Wang, Adaptive Fuzzy Systems and Control-Design and Stability Analysis, Prentice-Hall, Englewood Cliffs, NJ, USA, 1994.

[17] J. Fei and J. Zhou, "Robust adaptive control of MEMS triaxial gyroscope using fuzzy compensator," IEEE Transactions on Systems, Man, and Cybernetics, Part B (Cybernetics), vol. 42, no. 6, pp. 1599-1607, 2012.

[18] Y. Fang, J. Fei, and T. Hu, "Adaptive backstepping fuzzy sliding mode vibration control of flexible structure," Journal of Low Frequency Noise, Vibration and Active Control, no. article 146134841876709, 2018.

[19] Y. Chu, J. Fei, and S. Hou, "Dynamic global proportional integral derivative sliding mode control using radial basis function neural compensator for three-phase active power filter,"
Transactions of the Institute of Measurement and Control, vol. 40, no. 12, pp. 3549-3559, 2017.

[20] J. Fei and C. Lu, "Adaptive sliding mode control of dynamic systems using double loop recurrent neural network structure," IEEE Transactions on Neural Networks and Learning Systems, vol. 29, no. 4, pp. 1275-1286, 2018.

[21] J. Fei and T. Wang, "Adaptive fuzzy-neural-network based on RBFNN control for active power filter," International Journal of Machine Learning and Cybernetics, 2018.

[22] K. Rajagopal, F. Nazarimehr, A. Karthikeyan, A. Srinivasan, and S. Jafari, "Fractional order synchronous reluctance motor: Analysis, Chaos control and FPGA implementation," Asian Journal of Control, vol. 20, 2017.

[23] I. Podlubny, Fractional Differential Equations, Academic Press, New York, NY, USA, 1999.

[24] D.-Y. Chen, W. L. Zhao, X. Y. Ma, and R. F. Zhang, "Nochattering sliding mode control chaos in Hindmarsh-rose neurons with uncertain parameters," Computers \& Mathematics with Applications, vol. 61, no. 10, pp. 3161-3171, 2011.

[25] J. Fei and C. Lu, "Adaptive fractional order sliding mode controller with neural estimator," Journal of the Franklin Institute, vol. 355, no. 5, pp. 2369-2391, 2018.

[26] R. Garrappa, "Predictor-corrector PECE method for fractional differential equations," MATLAB Central File Exchange [File ID: 32918], 2012.

[27] K. Diethelm, N. J. Ford, and A. D. Freed, "A predictorcorrector approach for the numerical solution of fractional differential equations," Nonlinear Dynamics, vol. 29, no. 1/4, pp. 3-22, 2002.

[28] H. Kawakami, "Bifurcation of periodic responses in forced dynamic nonlinear circuits: computation of bifurcation values of the system parameters," IEEE Transactions on Circuits and Systems, vol. 31, no. 3, pp. 248-260, 1984.

[29] H. Kawakami, "Bifurcation and chaotic state in forced oscillatory circuits containing saturable inductors," International Journal of Applied Electromagnetics in Materials, vol. 3, pp. 215-220, 1992.

[30] K. Diethelm, The Analysis of Fractional Differential Equations: an Application-Oriented Exposition Using Differential Operators of Caputo Type, Springer, 2010.

[31] Y. Zhou, J. Wang, and L. Zhang, Basic Theory of Fractional Differential Equations, World Scientific, 2016.

[32] D. Baleanu, K. Diethelm, E. Scalas, and J. J. Trujillo, Fractional Calculus: Models and Numerical Methods. Series on Complexity, Nonlinearity and Chaos: Volume 3, World Scientific, 2016.

[33] D. Xue, C. Zhao, and Y. Chen, "A modified approximation method of fractional order system," in 2006 International Conference on Mechatronics and Automation, pp. 1043-1048, Luoyang, Henan, China, June 2006.

[34] D. Baleanu and S. I. Muslih, "Nonconservative systems within fractional generalized derivatives," Journal of Vibration and Control, vol. 14, no. 9-10, pp. 1301-1311, 2008.

[35] C. Chen, F. Liu, and K. Burrage, "Finite difference methods and a Fourier analysis for the fractional reaction-subdiffusion equation," Applied Mathematics and Computation, vol. 198, no. 2, pp. 754-769, 2008.

[36] T. A. M. Langlands and B. I. Henry, "The accuracy and stability of an implicit solution method for the fractional diffusion 
equation," Journal of Computational Physics, vol. 205, no. 2, pp. 719-736, 2005.

[37] F. Mainardi, A. Mura, G. Pagnini, and R. Gorenflo, "Subdiffusion equations of fractional order and their fundamental solutions," Mathematical Methods in Engineering, 2007.

[38] C. L. MacDonald, N. Bhattacharya, B. P. Sprouse, and G. A. Silva, "Efficient computation of the Grünwald-Letnikov fractional diffusion derivative using adaptive time step memory," Journal of Computational Physics, vol. 297, pp. 221-236, 2015.

[39] S. B. Yuste and L. Acedo, "On an explicit finite difference method for fractional diffusion equations," 2003, arxiv:cs/ 0311011.

[40] M. F. Tolba, A. M. AbdelAty, N. S. Soliman et al., "FPGA implementation of two fractional order chaotic systems," AEU - International Journal of Electronics and Communications, vol. 78, pp. 162-172, 2017.

[41] U. Feudel and C. Grebogi, "Multistability and the control of complexity," Chaos, vol. 7, no. 4, pp. 597-604, 1997.

[42] S. Kraut, U. Feudel, and C. Grebogi, "Preference of attractors in noisy multistable systems," Physical Review E, vol. 59, no. 5, pp. 5253-5260, 1999.

[43] S. Kraut and U. Feudel, "Multistability, noise, and attractor hopping: the crucial role of chaotic saddles," Physical Review E, vol. 66, no. 1, 2002.

[44] K. Rajagopal, P. Duraisamy, R. Weldegiorgis, and A. Karthikeyan, "Multistability in horizontal platform system with and without time delays," Shock and Vibration, vol. 2018, Article ID 1092812, 8 pages, 2018.

[45] A. Wolf, J. B. Swift, H. L. Swinney, and J. A. Vastano, "Determining Lyapunov exponents from a time series," Physica D: Nonlinear Phenomena, vol. 16, no. 3, pp. 285-317, 1985. 


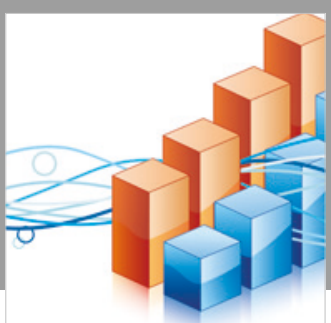

Advances in

Operations Research

\section{-n-m}
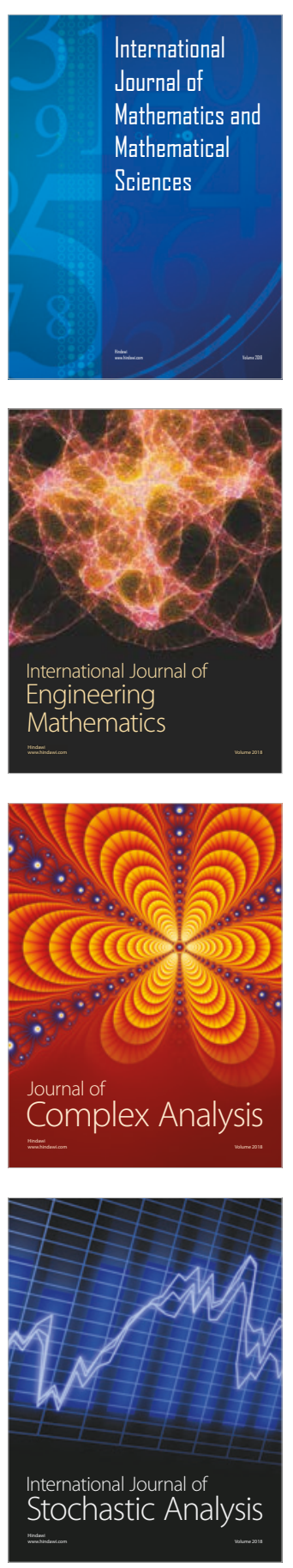
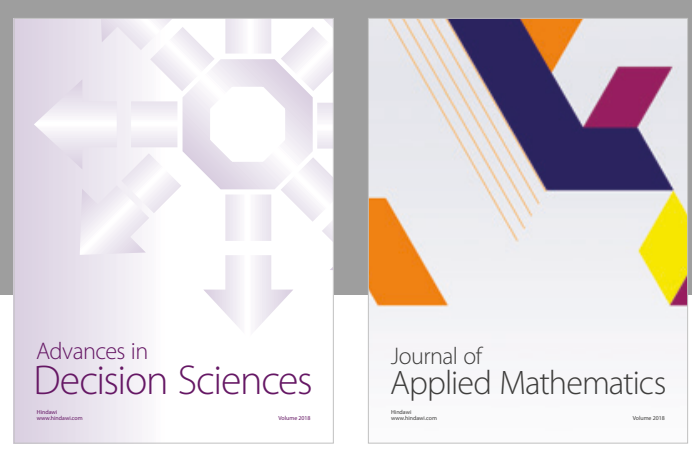

Journal of

Applied Mathematics
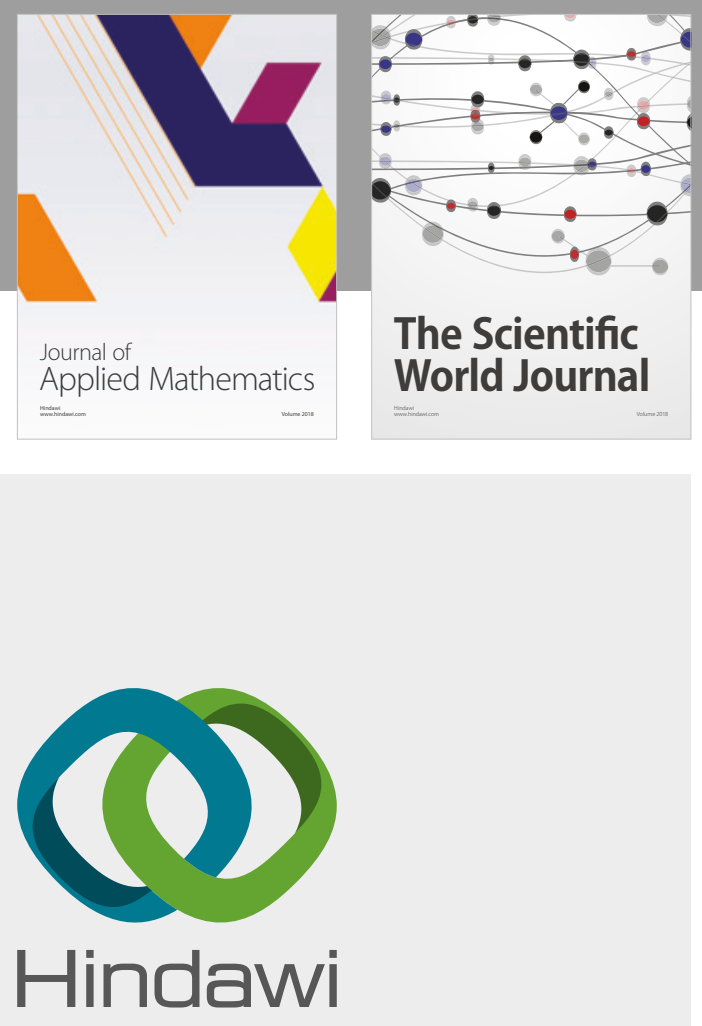

Submit your manuscripts at

www.hindawi.com

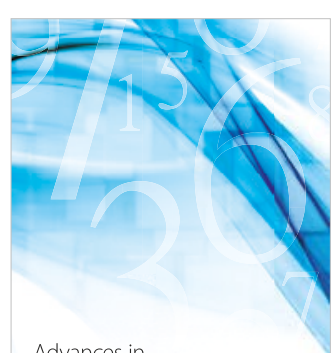

Advances in
Numerical Analysis
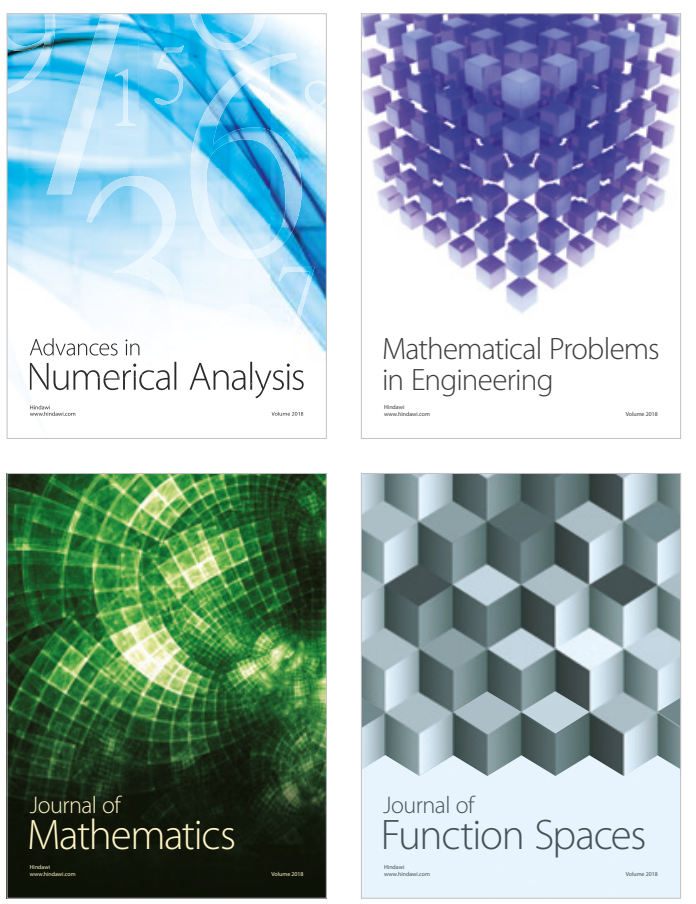

Mathematical Problems in Engineering

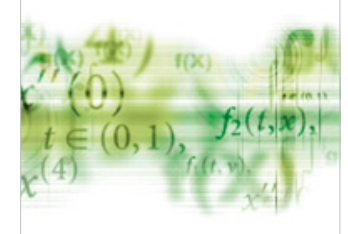

International Journal of

Differential Equations

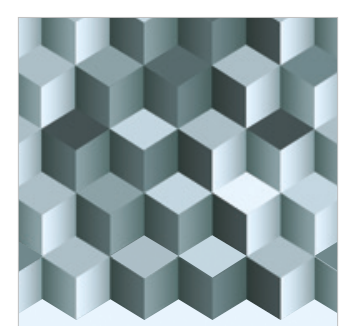

Journal of

Function Spaces
The Scientific

World Journal

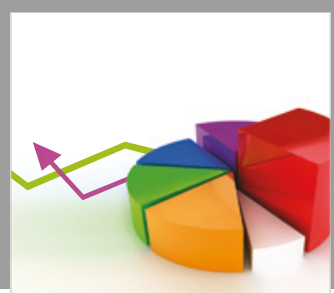

Journal of

Probability and Statistics
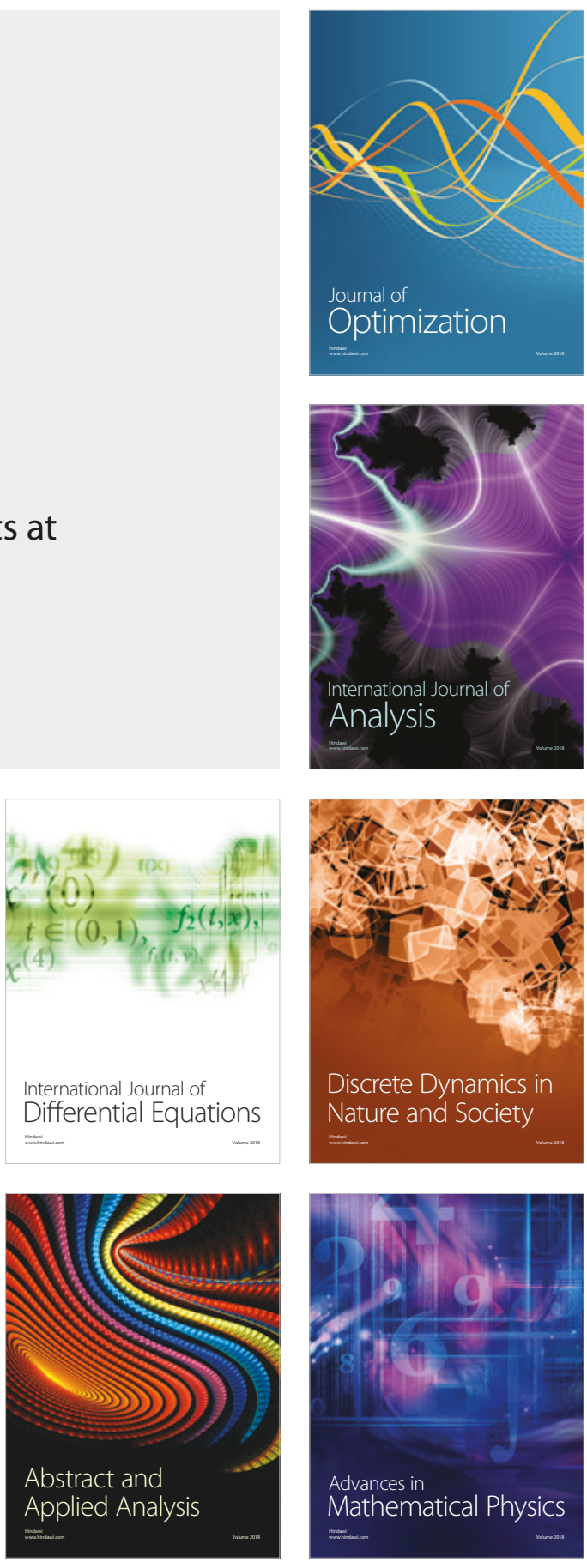